\title{
Establishing priorities in neurofibromatosis research: A workshop summary
}

\author{
Mia MacCollin, $M D^{1}$, David H. Gutmann, $M D$, PhD ${ }^{2}$, Bruce Korf, $M D, P h D^{3}$, and Robert Finkelstein, $P h D^{4}$
}

\begin{abstract}
Progress in understanding the biology of the neurofibromatoses (NF1 and NF2) offers hope for the development of new, effective methods of treatment. In May 2000, the National Institute of Neurological Disorders and Stroke (NINDS) hosted a workshop that included leading researchers and clinicians from the NF community. The goal of the meeting was to assess current knowledge and identify priorities for future research. Needs identified included the development of better animal models, further study of the function of the NF1 and NF2 genes, and investigation of the role of modifier genes. The participants agreed that it will also be important to define further the natural history of NF1 and NF2 and to develop an infrastructure to support clinical trials. They also discussed the possible creation of research consortia and NF centers to promote the integration of basic and clinical research.
\end{abstract}

During the past decade, researchers have made remarkable strides toward understanding neurofibromatosis (NF). This progress began with the isolation of the genes that cause NF1 and NF2, the two major forms of this neurogenetic disorder. Identification of these genes led to insights about disease mechanisms, the development of animal models, and the formulation of preliminary strategies for therapeutic intervention.

In May 2000, the National Institute for Neurological Disorders and Stroke (NINDS) hosted a workshop in Bethesda, Maryland, intended to assess the status of NF research and to facilitate future research efforts. In addition to a panel of 17 basic scientists and clinicians, the meeting included representatives from five other NIH Institutes (NCI, NICHD, NEI, NHLBI, and NIDCD), the Department of Defense, the Veteran's Administration, the National NF Foundation (NNFF), NF Inc., and Capitol Associates.

The meeting was divided into three sessions (Basic Science, Therapeutics, and Research Strategies), chaired by Luis Parada (University of Texas Southwestern Medical Center), Mia MacCollin (Massachusetts General Hospital), and Bruce Korf (Harvard Medical School), respectively. In each session, recent research results or proposals regarding research priorities were presented, followed by a discussion of their implications. In this report, we summarize the highlights of the meeting and

\footnotetext{
From the ${ }^{1}$ Neuroscience Center MGH East, Charlestown, Massachusetts; ${ }^{2}$ Washington University School of Medicine, Department of Neurology, Neurofibromatosis Program, St. Louis Children's Hospital, St. Louis, Missouri; ${ }^{3}$ Partners Center for Human Genetics, Boston, Massachusetts; and ${ }^{4}$ NINDS, NSC, Bethesda, Maryland.

Robert Finkelstein, PhD, NINDS, NSC Room 2143, 6001 Executive Blvd., Bethesda, MD 20892-9527.
}

present recommendations made by the participants for future initiatives.

\section{SESSION 1: BASIC SCIENCE}

It was agreed that the ability to develop targeted therapies for NF1 and NF2 depends upon an improved understanding of the molecular biology of the NF1 and NF2 gene products. Determining the function of the NF1 protein (neurofibromin) and the NF2 protein (merlin/schwannomin) will provide a foundation for designing mechanistically based, NF-specific therapeutic strategies. Insights into the mechanisms of action of these tumor suppressors will also broaden our understanding of cell growth control, which is important not only for understanding the neurofibromatoses, but other diseases as well.

Panelists presented several strategies for investigating the functions of neurofibromin and merlin. These included molecular, cellular, and organismal biological approaches.

\section{Molecular and cellular biology of neurofibromin and merlin}

Dr. David Gutmann discussed studies aimed at understanding how merlin forms intramolecular complexes required for its function as a negative growth regulator. He proposed that merlin exists in two conformations: an open, growth-permitting (inactive) form, and a closed growth-suppressing (active) form. The ability of merlin to assume these conformations may dictate its ability to interact with proteins that transduce merlin's growth suppressive signal.

Dr. Nancy Ratner described experiments involving one of the most common malignant tumors in NF1, the malignant peripheral nerve sheath tumor (MPNST). She and Dr. Jeffrey DeClue have shown that the epidermal growth factor receptor (EGF-R), which is not normally expressed in Schwann cells, is expressed in transformed Schwann cells from Nf1-deficient mice as well as in human MPNSTs. This result suggests that augmented growth factor receptor activity cooperates with loss of neurofibromin in the transformation of Schwann cells, an effect that may be relevant to MPNST pathogenesis. This observation also suggests possible therapeutic interventions involving the EGF-R pathway for MPNSTs.

\section{Organismal biology of neurofibromin and merlin}

Drs. Luis Parada and Tyler Jacks described recent work from their laboratories aimed at generating mouse models for NF1 by targeted disruption of the mouse $N f 1$ and p53 tumor suppressor genes. Both groups found that mice heterozygous for 
Nf1 and p53 mutations develop tumors that histologically resemble the MPNSTs seen in NF1 patients. Previous studies had shown that, in humans, NF1-associated MPNSTs harbor inactivating mutations in the $\mathrm{p} 53$ gene. This genetic cooperativity suggests that loss of both $N f 1$ and p53 expression is critical to the development of these malignancies in NF1 and the possibility that therapeutic interventions could be targeted to $\mathrm{p} 53$.

Dr. Nancy Ratner described her laboratory's intriguing results involving wound healing in $N f 1$ heterozygous ( $N f 1 \pm$ ) mice. When Nf1 \pm mice sustain a specific nerve injury, they display abnormal wound healing, develop skin pigmentation around the site of injury, and occasionally manifest tumors with some similarities to the benign neurofibromas observed in NF1 patients. These results point to a role for NF1 in wound healing.

Dr. David Gutmann described results from his laboratory on the effect of Nf1 heterozygosity on astrocyte proliferation. He showed that reduced, but not absent, neurofibromin expression in mice causes increased astrocyte proliferation both in vitro and in vivo. This finding suggests that some of the nontumor phenotypes observed in patients with NF1 may result from reduced, but not absent, neurofibromin expression.

Drs. Andre Bernards and Yi Zhong described elegant studies of the Drosophila Nf1 gene. Dr. Bernards isolated the fly gene and demonstrated that it is strikingly similar in sequence to the human NF1 gene. He then disrupted the gene, thereby generating flies lacking neurofibromin. These mutant flies are 20\% smaller than their wild-type counterparts. The size defect was not modified by manipulating Ras signaling, but was rescued by increasing, and enhanced or mimicked by decreasing signaling through the adenylyl cyclase (AC)-protein kinase A (PKA) pathway. A human NF1 transgene rescued the fly Nf1 mutant size defect, and preliminary evidence suggests that rasGAP activity is required for rescue. Therefore, the NF1 protein either has separate Ras and AC/PKA modulating activities, or defects in Ras pathways are upstream of AC/PKA signaling defects. Finally, Dr. Bernards described experiments using the fly to identify genes that cooperate with loss of neurofibromin to specify particular cellular defects. These other genes "modify" the effect of neurofibromin loss and could have human homologues that determine whether a particular individual develops an optic pathway glioma or a bony abnormality.

Dr. Zhong demonstrated that flies lacking neurofibromin have abnormal neuromuscular transmission as a result of defective PKA signaling. These Nf1-deficient flies also have subtle behavioral abnormalities in learning and memory. Dr. Zhong and his colleagues are trying to dissect the signaling pathways in the fly that are important for learning and memory. These studies may elucidate the pathways disrupted in NF1 patients with learning disabilities.

\section{Priorities for future basic research}

The panelists agreed upon several research priorities. These priorities included the development of more refined animal models for NF1 and NF2, the further analysis of the mecha- nisms of action of neurofibromin and merlin, and the identification of modifier genes.

\section{Refined animal models of NF1 and NF2}

It was agreed that developing genetically refined and tissuespecific animal models is a high research priority. Genetically refined models include those in which cooperating genes are introduced into existing animal models to determine the interplay between neurofibromin/merlin and other proteins important in growth regulation. Tissue-specific models in which neurofibromin/merlin is disrupted in astrocytes, neurons, and other cells will provide important insights into the role of neurofibromin/merlin in specific tissues and may provide tissuespecific preclinical models. The effect of heterozygosity deserves further exploration in that some of the clinical features of NF1 and NF2 may not result from total absence of neurofibromin/merlin expression. Lastly, the function of neurofibromin and merlin in specific tissues may reflect a particular developmental or cellular context. In this regard, loss of neurofibromin or merlin may result in tumor formation only in a particular cellular environment or during a specific developmental time frame. The development of animal models for $\mathrm{NF} 1$ and NF2 that permit $N f 1$ and $N f 2$ inactivation under these different conditions may provide insights into why some NF1 and NF2 tumors grow in an unpredictable fashion.

\section{Function of neurofibromin and merlin}

Further studies are required to determine the function of these two critical growth regulators. Although neurofibromin has been shown to function as a rasGAP, studies using Drosophila suggest that the PKA pathway may also be affected by neurofibromin loss. In addition, multiple molecules are activated by ras. The effect of neurofibromin deficiency on the activity of these downstream molecules in specific tissues may provide additional targets for therapeutic intervention. Finally, the function of the $90 \%$ of the neurofibromin protein that lies outside the rasGAP domain is unknown. Future work may elucidate tissue-specific neurofibromin protein interactions involving this region. Further work also needs to be done to identify the mechanism of action of merlin. Of particular importance will be the identification and analysis of proteins that interact with merlin.

\section{Modifier genes}

Further work to identify genes that modify the phenotypic expression of neurofibromin and merlin is essential. The use of more malleable systems, such as Drosophila, may uncover some of these genes. Studies using both the mouse and the fruit fly will be crucial, given the inability of present genetic testing to provide predictive information.

\section{SESSION 2: THERAPEUTICS}

The goal of this session was to determine how the development of rational therapeutics for NF can be facilitated. The panelists discussed current therapeutic strategies and identi- 
fied key issues that must be resolved before successful pharmacological treatment of NF can be achieved. Discussion focused primarily on the treatment of tumors associated with NF. Although learning disabilities, skeletal malformations, and other symptoms can be of equal clinical importance, they may require a fundamentally different therapeutic approach.

\section{Natural history issues and outcome assessment}

The development of successful therapeutic interventions for NF will depend on a detailed understanding of phenotype and natural history (Table 1). ${ }^{1-6}$ A number of features make NF unique among neoplastic diseases, including (1) the presence of both tumorous and nontumorous manifestations, which may have distinct pathogenetic mechanisms and natural histories; (2) the unpredictability of growth rate of NF-related tumors, which can include cessation of growth without treatment; and (3) the wide range of variability of phenotypic expression among patients.

Much of our knowledge of the natural history of NF1 is based on clinical observations made decades ago. Although important, these observations lack the depth and detail that can be obtained using modern methodologies, such as molecular genetics and magnetic resonance imaging. Improved assessment will be imperative to judge the outcomes of treatment trials. The following were identified as major needs to permit more sophisticated outcome assessment:

1. Developing a standardized set of tools for clinical documentation (including a phenotypic database), reliable mechanisms for measuring tumor growth, and standardized markers of functional impairment.

2. Obtaining a better understanding of the role of the NF genotype and modifier genes in the variable expressivity of NF.

Table 1

Major tumor manifestations of NF

\begin{tabular}{lcc}
\hline Feature & \% Affected & \% Requiring intervention \\
\hline NF1 & 100 & 25 \\
Cutaneous tumors & 33 & 8 \\
Plexiform neurofibromas & 5 & 5 \\
MPNST & 15 & 1.5 \\
Optic pathway changes & & \\
& & \\
NF2 & 100 & 90 (estimated) \\
Vestibular schwannoma & 50 & 25 (estimated) \\
Meningioma & 75 & 25 \\
Spinal cord tumor &
\end{tabular}

At this time, intervention for all tumor types is primarily surgical. Note that MPNST in NF1 is uniformly fatal if not treated. Data derived from Evans et al., ${ }^{1}$ Huson et al., ${ }^{2}$ Listernick et al., ${ }^{3}$ Mautner et al., ${ }^{4}$ Parry et al., ${ }^{5}$ Thakker et al., ${ }^{6}$ and M. MacCollin, personal observations.
3. Establishing an infrastructure to support clinical trials for NF.

\section{Current therapeutic approaches}

Recent advances in our understanding of signal transduction, apoptosis, cell cycle regulation, angiogenesis, and other cellular processes are providing a basis for developing anticancer pharmaceuticals. Jackson Gibbs (Merck) summarized progress in this area. An increasing number of clinical trials involve drugs targeted to proteins in specific signal transduction cascades. For example, promising results are being obtained with a small molecule inhibitor of bcr-abl kinase (for $\mathrm{CML}$ ) and with inhibitors of the EGF receptor. Further research will be necessary to reveal if these and other inhibitors can affect NF-associated tumors.

Several approaches have been taken to modulate the Ras signaling pathway, which is activated in NF1 tumors. Among the reagents being tested are farnesyl transferase inhibitors and Ha-ras and Raf antisense constructs. Small molecule inhibitors directed against Raf and MEK are also being developed. Other agents in development that should be considered for NF tumors include inhibitors of cyclin-dependent kinases and Onyx-015 (a p53 based therapeutic), which is showing activity in head and neck cancers and in liver metastases. It will also be important to determine whether antiangiogenesis inhibitors are effective in blocking NF tumor growth. Finally, trials have shown that metalloproteinase inhibitors are not more effective than existing therapeutics, but this finding may be due to clinical trial design.

\section{Clinical trials involving NF patients}

Specific drugs have been tested in clinical trials that have included NF patients, and other agents have been suggested for future trials based on their mechanism of action (Table 2). There are indications that tumor responsiveness may differ in NF patients as compared to patients with single sporadic tumors, but this issue has not been systematically studied. For example, in optic nerve glioma, radiation therapy may cause a higher incidence of sequelae in NF patients. For other NFassociated tumors, such as plexiform neurofibromas, treatment options are very limited. There is some clinical evidence that drugs such as alpha-interferon and thalidomide may provide symptomatic relief and occasionally cause tumor shrinkage. A major limitation of the study of patients with progressive plexiform neurofibromas who undergo treatment is the lack of an infrastructure to perform such studies.

The panelists agreed that treatment endpoints for clinical trials involving patients with plexiform neurofibromas and other NF-associated tumors must be redefined. Although linear measures of tumor growth will continue to be a criterion for drug efficacy, such measurements are not easily determinable for slow-growing tumors with irregular margins. Volumetric analyses of tumor size are more precise but cumbersome to determine and of unproven utility. The ultimate standard of treatment efficacy should probably be the time to clinical progression, but this is a subjectively defined parame- 
Table 2

Possible pharmacological agents for NF1 and NF2

\begin{tabular}{ll}
\hline $\begin{array}{l}\text { Clinical trials that have included NF1 } \\
\text { patients }\end{array}$ & $\begin{array}{c}\text { Clinical trials that have included } \\
\text { NF2 patients }\end{array}$ \\
Retinoic acid & Hydroxyurea \\
Interferon & RU486 \\
Thalidomide & Antineoplastin \\
Optic nerve glioma & Interferon alpha \\
FTI (R115777) & Temozolomide \\
MPNST protocols & \\
Ketotifen &
\end{tabular}

Agents proposed for NF1

Agents proposed for NF2

Other antiangiogenesis agents

Other Ras pathway inhibitors

Quinidine

Gene or protein product replacement

Although there is no accepted pharmacological treatment for NF1 or NF2, several clinical trials have focused on or included NF patients and additional pharmacological agents have been proposed based on cell or molecular biological results.

ter that varies according to tumor location. Another important factor is improvement in quality of life, and this issue should be evaluated in future trials. In certain cases, surrogate markers will be useful, particularly when therapeutic reagents are used that affect known cellular processes. For example, vascular endothelial growth factor assays could be used for evaluating antiangiogenesis agents, and measures of cellular proliferation employed for testing drugs that block signal transduction cascades (e.g., farnesyl transferase inhibitors).

\section{Gene therapy for NF}

Gene therapy, although at an early stage of development, offers promise for the treatment of NF lesions. The most promising initial targets would be overgrowths that are accessible to direct injection with vectors and that are otherwise inoperable or life/function threatening. Such overgrowths could include plexiform neuromas, spinal meningiomas, and vestibular schwannomas. Vectors that do not express viral genes and, therefore, cause minimal toxicity and immunoreactivity should be used. These include retrovirus/lentivirus, adenoassociated virus (AAV), "gutted" adenovirus, and helper virusfree HSV-1 amplicon vectors. Hybrid vectors, such as the HSV/ Epstein Barr Virus (EBV)/retrovirus amplicons, also allow direct conversion of tumor cells to retrovirus packaging cells, thus expanding the range of gene delivery.

The key issue for gene therapy will be the selection of appropriate therapeutic genes. Genes being tested against malignant tumors frequently target angiogenesis or rapid cell division and may not be appropriate for many NF-associated lesions. In addition, even after multiple injections, gene delivery will generally be limited to a small region of a tumor. The therapeutic efficacy of a particular gene product will be amplified if it can act in a nonautonomous fashion. For example, proteins that induce the release of secreted factors (that, for example, promote apoptosis) may be effective outside the site(s) of injection.

\section{Using animal and cell culture models to test potential therapies}

Given the difficulties involved in performing clinical trials and measuring efficacy in NF patients, murine models provide attractive systems for preclinical testing of rational therapeutics. Collaborations involving the Shannon, Jacks, Parada, Copeland, and Jenkins laboratories resulted in the production of mice with a myeloproliferative disorder (MPD) closely resembling JMML, caused by the injection of Nf1-deficient fetal liver cells into irradiated hosts. Children with NF1 are strongly predisposed to JMML.

A recent set of experiments using Nf1 mutant mice addressed the general question of how abnormal responsiveness to growth factors might contribute to the pathologic features of NF1. Because of in vitro data implicating GM-CSF in the development of JMML, the Shannon laboratory crossed strains of GM-CSF and NF1 mutant mice. This study showed that hypersensitivity to a specific growth factor plays a central role both in establishing and maintaining the aberrant growth of NF1 mutant hematopoietic cells. These findings raise the possibility that growth factors that act upon other tissues could contribute to the pathogenesis of other malignant and nonmalignant complications of NF1, and may, therefore, be appropriate targets for therapeutic intervention.

This mouse model of JMML is promising for preclinical testing of anti-Ras pathway therapeutics for several reasons. These include the subacute nature of the MPD that arises in the immunocompetent recipients of Nf1-deficient fetal liver cell transplants and the central role of hyperactive Ras in this and other human malignancies. Perhaps most importantly, it will be possible to perform biochemical studies on primary hematopoietic cells to determine directly whether experimental therapeutics interact with their intended molecular targets. For example, the Shannon laboratory recently used this model to evaluate the effects of the farnesyl transferase inhibitor (FTI) L-744,832 on specific parameters of Ras signaling in vivo and in vitro.

A major challenge has been to develop better models for neurofibroma, sarcoma, vestibular schwannoma, meningioma, and astrocytoma. The laboratories of Drs. Jacks and Parada utilized mice lacking both $\mathrm{p} 53$ and Nf1 in cis to produce a reproducible sarcoma model, and many cell lines have been generated that will facilitate the screening of promising agents. The Jacks lab also produced neurofibroma-like lesions in chimeras constructed by injecting Nf1-deficient ES cells into wildtype blastocysts. Finally, the production of strains of mice in which inactivation of $\mathrm{Nf} 1$ or $\mathrm{Nf} 2$ can be restricted to specific tissues and/or induced during a specific time window holds great promise.

The Department of Defense recently approved a proposal to form a Consortium to enhance existing murine models of NF1- and NF2-associated tumors, and to develop new strains. 
These mice will be valuable for preclinical testing of compounds that might provide benefit to patients with NF1 and NF2. The participants in the NINDS meeting generally favored this idea, and there was extensive discussion of the challenges involved in modeling neurofibroma, astrocytoma, and other $\mathrm{NF}$-associated lesions. It was also agreed that developing in vitro cell culture models that could be used for high throughput drug testing should be a priority.

\section{SESSION 3: RESEARCH STRATEGIES}

As discussed in the previous two sessions, progress in understanding the biology of NF1 and NF2, as well as advances in neurobiology and cancer research in general, offer hope for the development of effective therapies. In this session, the panelists discussed funding strategies that could accelerate the pace of basic research, the development of therapeutics, and the performance of clinical trials.

To date, most of our understanding of the basic biology of NF1 and NF2 has derived from investigator-initiated research funded either by the NIH or the Department of Defense. The Department of Defense has also funded natural history studies of both NF1 and NF2. Much of the research needed to advance our approach to the neurofibromatoses, however, is not hypothesis-driven; this includes the development of animal models, identification of genotype-phenotype correlations, search for modifying genes, investigation of natural history, and development of outcomes measurements. Thus, while traditional investigator-initiated funding will be important to continue progress in understanding of basic biology, a number of additional funding mechanisms will be needed.

Two complementary approaches would be to fund NF Research Consortia and NF Centers. The consortia would consist of groups of laboratories or clinical programs that would work together to accomplish a goal such as the development of animal models or clinical assessment tools. NF Centers would be collaborations of one or more clinical programs and research laboratories that would provide care to a group of patients and utilize this resource to investigate the biology of NF1 and/or NF2 in collaboration with research laboratories. The consortia, therefore, would have specific missions aimed at developing resources needed by the NF community; the NF Centers would concentrate clinical and research resources aimed at providing a more comprehensive approach to clinical and basic research.

\section{Consortia}

The panelists proposed that multicenter consortia be established to develop resources needed to facilitate the development and testing of new therapies. These would include (1) the development of animal models and preclinical drug testing schemes; (2) the development of in vitro assays and large scale drug screening schemes; and (3) the development of standards for clinical trials, including measurement tools for tumors, a database system for recording clinical information, and methods for assessing functional impairment.

\section{NF centers}

It was proposed that programs be established that combine one or more strong clinical centers with one or more research laboratories. The goal would be to provide comprehensive care, conduct clinical research, and systematically investigate aspects of the pathogenesis of NF1 and NF2. These centers would not necessarily be geographically based; rather, a strong clinical program could group with one or more additional clinical programs and research laboratories that might be geographically distributed but would be linked together by a common clinical approach and a set of related research questions. A number of such centers distributed around the country would develop highly concentrated clinical expertise, which would attract patients and make clinical material available for research use.

\section{CONCLUSION}

At the present time, there are remarkable opportunities for basic and clinical researchers investigating the neurofibromatoses. The isolation of the NF1 and NF2 genes, the preliminary discoveries regarding their function, the development of animal models, and other advances have begun to provide the tools for the development of rational therapeutic approaches. To capitalize on this opportunity, the panelists agreed that the results of this workshop would be discussed with the broader NF research community at the national meeting in Aspen, Colorado.

\section{PANELISTS}

We gratefully acknowledge the contributions of the panelists who participated in this workshop. They included Andre Bernards (MGH Cancer Center; Charlestown, MA), Xandra Breakefield (MGH East; Charlestown, MA), Jonathan Epstein (University of Pennsylvania School of Medicine; Philadelphia, PA), Jackson Gibbs (Merck; West Point, PA), James Gusella (MGH; Charlestown, MA), Tyler Jacks (MIT; Cambridge, MA), Frank McCormick (UCSF; San Francisco, CA), Roger Packer (Children's National Medical Center; Washington, DC), Luis Parada (University of Texas Southwestern Medical Center; Dallas, TX), Nancy Ratner (University of Cincinnati College of Medicine; Cincinnati, $\mathrm{OH}$ ), Allan Rubenstein (Mt. Sinai School of Medicine; New York, NY), Kevin Shannon (UCSF; San Francisco, CA), William Slattery (House Ear Institute; Los Angeles, CA), Bruce Stillman (CSH Laboratory; Cold Spring Harbor, NY), and Yi Zhong (CSH Laboratory; Cold Spring Harbor, NY).

\section{Acknowledgments}

We thank Phillip Sheridan (FDA) for his important role in co-organizing the workshop and Lorraine Fitzsimmons (NINDS) for many helpful suggestions, and we are grateful to representatives of NCI, NICHD, NEI, NHLBI, and NIDCD for 
assistance and to the NIH Office of Rare Diseases and NHLBI for financial support.

\section{References}

1. Evans DGR, Huson S, Donnai D, Neary W, Blair V, Newton V, Harris R. A clinical study of type 2 neurofibromatosis. Q J Med 1992;84:603-618.

2. Huson SM, Harper PS, Compston DAS. von Recklinghausen Neurofibromatosis. A clinical and population study in South-East Wales. Brain 1988;111:1355-1381.
3. Listernick R, Louis D, Packer R, Gutmann D. Optic pathway gliomas in children with neurofibromatosis 1: consensus statement from the NF1 optic pathway glioma task force. Ann Neurol 1997;41:143-149.

4. Mautner VF, Lindenau M, Baser M, Hazim W, Tatagiba M, Haase W, Samii M, Wais R, Pulst SM. The neuroimaging and clinical spectrum of neurofibromatosis 2. Neurosurgery 1996;38:880-886.

5. Parry DM, Eldridge R, Kaiser-Kupfer MI, Bouzas EA, Pikus A, Patronas N. Neurofibromatosis 2 (NF2): clinical characteristics of 63 affected individuals and clinical evidence for heterogeneity. Am J Med Genet 1994;52:450-461.

6. Thakkar SD, Feigen U, Mautner VF. Spinal tumours in neurofibromatosis type1: an MRI study of frequency, multiplicity and variety. Neuroradiology 1999;41:625-629. 